\title{
EQUATORIAL ZONAL JETS AND JUPITER's GRAVITY
}

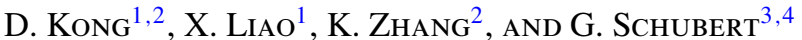 \\ ${ }^{1}$ Key Laboratory of Planetary Sciences, Shanghai Astronomical Observatory, Chinese Academy of Sciences, Shanghai 200030, China \\ ${ }^{2}$ Center for Geophysical and Astrophysical Fluid Dynamics, University of Exeter, Exeter, EX4 4QE, UK \\ ${ }^{3}$ Department of Earth, Planetary, and Space Sciences, University of California, Los Angeles, CA 90095-1567, USA \\ Received 2014 June 19; accepted 2014 July 17; published 2014 August 1
}

\begin{abstract}
The depth of penetration of Jupiter's zonal winds into the planet's interior is unknown. A possible way to determine the depth is to measure the effects of the winds on the planet's high-order zonal gravitational coefficients, a task to be undertaken by the Juno spacecraft. It is shown here that the equatorial winds alone largely determine these coefficients which are nearly independent of the depth of the non-equatorial winds.
\end{abstract}

Key words: gravitation - planets and satellites: atmospheres - planets and satellites: gaseous planets

\section{INTRODUCTION}

Alternating, fast cloud level zonal winds on Jupiter have been measured for several decades (Limaye 1986; Atkinson et al. 1996; Porco et al. 2003) but their depth of penetration into the Jovian interior, which is closely associated with the origin of the winds, still remains controversial. The Juno spacecraft will probe the depth of penetration of the zonal winds by measuring their effects on the high-order zonal gravitational coefficients at unprecedentedly high precision (Bolton 2005). Here we show that the equatorial winds alone largely determine the high-order gravitational coefficients. The equatorial region of Jupiter is dynamically special because its rapid rotation exerts the strongest control on the fluid motion there through the largest boundary curvature (Zhang 1993). Magnetic braking and stable stratification, as confirmed by direct numerical simulations of Jovian interior dynamics (Jones \& Kuzanyan 2009; Gastine \& Wicht 2012), have negligible effects on the equatorial zonal winds that extend unimpeded through Jupiter in the direction parallel to the rotation axis. The gravitational field is also sensitive to the equatorial region because of its location in non-spherical Jupiter. Based on an oblate spheroidal-shell model of a polytropic Jupiter with index unity (Chandrasekhar 1933; Hubbard 1974, 1999), we calculate, using a fully three-dimensional finite-element method, the gravitational signature produced by the Jovian equatorial zonal winds that are equatorially symmetric and confined within a small equatorial region between latitudes $\varphi= \pm 25^{\circ}$ with maximum penetration depth about $10 \%$ of Jupiter's equatorial radius and containing about $0.18 \%$ of the Jovian mass. It is found, by comparing to results from a model of the deep zonal winds at all latitudes, that the equatorial zonal jets with $0.18 \%$ of the Jovian mass contribute $90 \%$ of the high-order gravitational coefficient $J_{12}$. Thus, the high-order gravitational coefficient-whose values are thought to reflect the penetration depth of the zonal winds-becomes nearly independent of the depth of the Jovian zonal winds in the non-equatorial regions with latitudes $25^{\circ} \leqslant \varphi \leqslant 90^{\circ}$ and $-90^{\circ} \leqslant \varphi \leqslant-25^{\circ}$.

The generation and maintenance of Jovian zonal winds has been a major scientific mystery in planetary sciences. There exist at least three different views on the origin of the winds. The first is that they are generated by thermal convection, partly powered by internal heat, which takes place within the deep interior of

\footnotetext{
4 Corresponding Author.
}

the planet (Busse 1976). The second is that the alternating zonal winds are primarily confined to a thin top layer of a stably stratified Jovian atmosphere (Ingersoll \& Cuzzi 1969; Lian \& Showman 2010). The third is that the winds are powered by internal heat via thermal convection, but they are confined within the planet's outermost thin shell, about $10 \%$ of Jupiter's radius (Heimpel et al. 2005), because the effect of magnetic braking by the Jovian magnetic field limits their depth of penetration (Liu et al. 2008). If the first and third views are right, the deep zonal winds of Jupiter generate an externally measurable gravitational signature by inducing substantial density anomalies (Hubbard 1999; Kaspi et al. 2010; Kong et al. 2013a). The Juno mission aims to resolve this long-term scientific puzzle about the depth of the zonal winds through its high-precision gravitational measurements together with accurate theoretical modeling.

Accurate modeling of the gravitational signature produced by the Jovian zonal winds is necessary for interpreting Juno's highprecision gravitational measurements. As a consequence of its rapid rotation, the shape and dominant zonal winds of Jupiter are primarily equatorially symmetric

$V_{g}(r, \theta)=-\frac{G M_{J}}{r}\left[1-\sum_{\operatorname{even} n}\left(J_{n}+\Delta J_{n}\right)\left(\frac{R_{e}}{r}\right)^{n} P_{n}(\cos \theta)\right]$ with $r \geqslant R_{e}$,

where $G=6.67384 \times 10^{-11} \mathrm{~m}^{3} \mathrm{~kg}^{-1} \mathrm{~s}^{-2}$ is the universal gravitational constant, $M_{J}=1.8986 \times 10^{27} \mathrm{~kg}$ is Jupiter's mass, $R_{e}=71492 \mathrm{~km}$ is the equatorial radius of Jupiter at the onebar surface (Seidelmann et al. 2007), $n$ takes even integers, $(r, \theta, \phi)$ are spherical polar coordinates with $\theta=0$ at the axis of rotation, $J_{2}, J_{4}, J_{6}, \ldots$, are the zonal gravitational coefficients of solid body rotation and $\Delta J_{n}$ represent the corrections caused by the zonal winds. $G M_{J}$ is known from orbital dynamics to greater accuracy while the Jovian mass $M_{J}$ represents a derived parameter. According to the Pioneer 11 data which give $\left(G M_{J}\right)_{P 11}=(12668667 \pm 324) \times 10^{9} \mathrm{~m}^{3} \mathrm{~s}^{-2}$ (Null 1976) together with a modern value for the gravitational constant $G=(6.674215 \pm 0.000092) \times 10^{-11} \mathrm{~m}^{3} \mathrm{~kg}^{-1} \mathrm{~s}^{-2}$ (Gundlach \& Merkowitz 2000), we obtain $M_{J}=\left(G M_{J}\right)_{P 11} / G=$ $1.8983 \times 10^{27} \mathrm{~kg}$. At present, only the first three zonal gravitational coefficients are accurately measured (Jacobson, JUP230 orbit solution, http://naif.jpl.nasa.gov/pub/naif/JUNO/kernels/ spk/jup230.bsp.lbl(2003)) but the Juno spacecraft (Bolton 2005) will perform high-precision measurements up to $n=12$. To accurately capture the Jovian gravitational signature caused solely by its deep zonal winds, one must take into account the full 


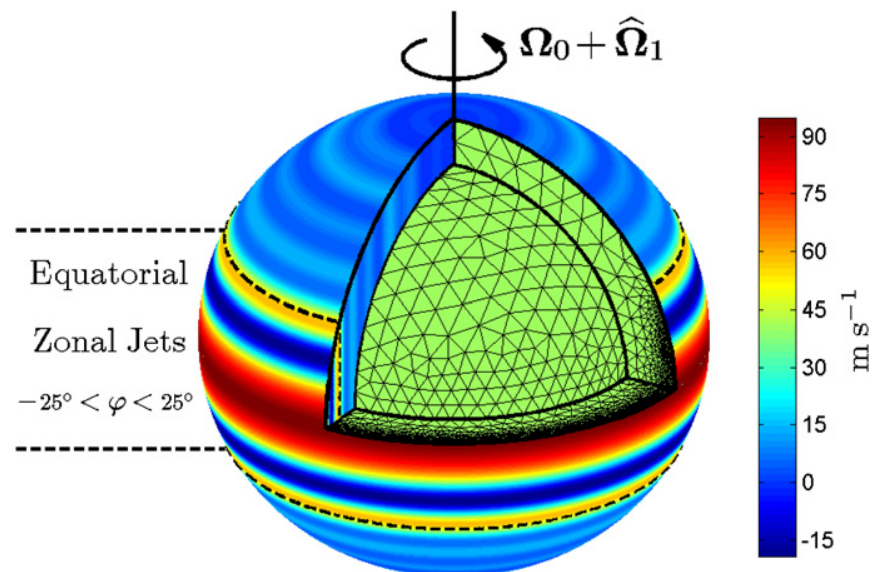

Figure 1. Schematic of Jovian zonal winds and the nonuniform, threedimensional finite-element mesh used in our numerical computation. Here $\Omega_{0}$ is the angular velocity of Jupiter's solid body rotation and $\widehat{\Omega}_{1}$ represents the angular velocity of its differential rotation. Red indicates eastward winds while blue denotes westward winds. The equatorial region enclosed by the dashed lines is defined as the domain between the latitudes $\varphi= \pm 25^{\circ}$ with the maximum depth $10 \%$ of the equatorial radius containing about $0.18 \%$ of the total Jovian mass. The nonuniform mesh has $32 \times 10^{6}$ tetrahedral elements within the outer molecular envelope and much denser nodes in the equatorial region where the zonal winds change rapidly and high numerical accuracy is required.

effect of rotational distortion to separate the winds signature $\Delta J_{n}$ from the measured values $\left(J_{n}+\Delta J_{n}\right)$.

Hubbard (1999) first analyzed the effect of deep zonal winds on Jupiter's gravitational harmonics in spherical geometry by assuming that Jupiter is in hydrostatic equilibrium with a polytropic index of unity and that the observed zonal winds extend parallel to the axis of rotation through the whole interior of the planet at all latitudes. His spherical analysis was extended by Kong et al. (2013a), who computed the gravitational coefficients of rotationally distorted Jupiter with non-spherical geometry (see also Kong et al. 2012). Both studies demonstrated that the contribution to the high-order coefficients $\left(J_{n}+\Delta J_{n}\right)$ like $\left(J_{12}+\Delta J_{12}\right)$ comes mainly from the effect of the zonal winds. By introducing an extra parameter $H$ describing the penetration depth of the zonal winds into the Jovian interior at all latitudes, Kaspi et al. (2010) constructed a spherical-shell model of the zonal winds and showed that, if the depth $H$ of the zonal winds is greater than $5 \times 10^{5} \mathrm{~m}$ everywhere, the variation of the gravity coefficient $\Delta J_{12}$ caused by the winds would be greater than the corresponding solid body value $J_{12}$. The coefficient $\left(J_{12}+\Delta J_{12}\right)$ is particularly important not only because it will be accurately measured by Juno but also because the gravitational harmonic with $n=12$ is just high enough to be directly associated with the winds. Their computational result (Kaspi et al. 2010) confirms that the gravity measurements would be sensitive to the depth $H$ of the internal zonal winds and, consequently, measuring the size of $\left(J_{12}+\Delta J_{12}\right)$ would help determine the penetration depth of the zonal winds into the interior.

\section{MODEL}

We define the Jovian equatorial region as a small domain between latitudes $\varphi= \pm 25^{\circ}$ with the maximum depth about $10 \% R_{e}$ in the equatorial plane containing about $0.18 \%$ of the Jovian mass $M_{J}$, as illustrated in Figure 1. For the rapidly rotating gaseous Jupiter whose eccentricity $\mathcal{E}_{J}$ at its one-bar surface (Seidelmann et al. 2007) is $\mathcal{E}_{J}=0.3543$, the equatorial region is dynamically special and gravitationally important. Fluid motion in the Jovian interior is dynamically controlled by its rapid rotation and strongly affected by the self-generated magnetic field, density stratification and boundary curvature. Since the equatorial region is marked by the largest boundary curvature with respect to the rotation axis, its rapid rotation exerts the strongest control there and helps generate a strong equatorial zonal flow. Jupiter possesses a large magnetic field whose dominant dipole tilts about $10^{\circ}$ to the rotation axis. It is generated by the magnetohydrodynamic dynamo process taking place primarily in its metallic conducting region $r<0.78 R_{J}$ because its molecular outer region $r>0.78 R_{J}$ has weak electric conductivity (Stevenson 1982). Since the equatorial winds are located in the non-metallic molecular region and since the Jovian dipolar field in the outer equatorial region is weak, magnetic braking associated with the Lorentz force (Liu et al. 2008) has a negligible effect on the equatorial zonal jets. Recent numerical computations (Jones \& Kuzanyan 2009; Gastine \& Wicht 2012) demonstrated that, while both stratification and magnetic braking strongly restrict the penetration depth of the zonal winds in middle and high latitudes, they have little influence in the equatorial region where the strong zonal winds extend unimpeded through the region in the direction parallel to the rotation axis. Since the zonal coefficients measure the departure of the density distribution from spherical symmetry, the high-order gravitational coefficients such as $J_{12}$ will be highly sensitive to the wind-induced density anomalies localized in the equatorial region.

Since the Jovian equatorial zonal winds are hardly affected by stratification and magnetic braking (Jones \& Kuzanyan 2009; Gastine \& Wicht 2012), this study focuses on the external gravitational signature produced by the equatorial jets. Jupiter is assumed to be isolated, rotating rapidly about its symmetry axis with the unit vector $\hat{\mathbf{z}}$, equatorially symmetric, and made of a fully compressible barotropic gas with a polytropic index of unity (Chandrasekhar 1933; Hubbard 1974). We have adopted the classic polytropic equation of state (EOS), instead of a more realistic EOS (Nettelmann et al. 2012), since the form of the EOS is unlikely to have a significant influence on the wind-induced density anomalies of the equatorial region. In an inertial frame, the fluid motion in the interior of Jupiter is assumed to comprise two major components (Hubbard 1999): (1) solid body rotation $\Omega_{0} \hat{\mathbf{z}} \times \mathbf{r}$ with angular velocity $\Omega_{0}=1.7585 \times 10^{-4} \mathrm{~s}^{-1}$ and $\mathbf{r}$ the position vector and (2) equatorially symmetric zonal winds $\widehat{\Omega}_{1} \hat{\mathbf{z}} \times \mathbf{r}$ with $\left|\widehat{\Omega}_{1} / \Omega_{0}\right| \ll 1$, where $\widehat{\Omega}_{1}$ denotes the angular velocity of differential rotation. To isolate the gravitational signature solely caused by zonal winds, we must consider two mathematically and physically coupled problems. In the first problem, we determine the non-spherical shape of Jupiter caused by its rapid rotation and compute its internal density distribution without zonal winds. Because the solution domain is a priori unknown, we adopt an iterative procedure, using a three-dimensional finite-element method, by adjusting an a priori unknown coefficient in the EOS until the model shape matches exactly the observed shape of Jupiter (Kong et al. $2013 b$ ). With both the shape and the density distribution, we then calculate the zonal gravitational coefficients $J_{2}, J_{4}, J_{6}, \ldots, J_{12}$ taking full account of rotation-induced shape change of Jupiter.

Our primary concern is, however, the second problem which determines the external gravitational signature of the equatorial zonal jets. According to Jovian models (Stevenson 1982; Guillot 2005), the interior, if the small rocky core is neglected, consists of two major parts: the metallic hydrogen-helium region 
Table 1

The Zonal Gravitational Coefficients up to $n=12$ Computed for $D=\infty, 0.1$ for an Oblate Spheroidal Jupiter with Eccentricity $\mathcal{E}_{J}=0.3543$

\begin{tabular}{lrcccc}
\hline \hline$n$ & $J_{n} \times 10^{6}$ & $\left(\Delta J_{n} \times 10^{6}\right)_{D=\infty}$ & $\left|\Delta J_{n} / J_{n}\right|_{D=\infty}$ & $\left(\Delta J_{n} \times 10^{6}\right)_{D=0.1}$ & $\left|\Delta J_{n} / J_{n}\right|_{D=0.1}$ \\
\hline 2 & +14167.271 & -1.401 & $0.010 \%$ & -1.355 & $0.010 \%$ \\
4 & -504.953 & +0.210 & $0.042 \%$ & +0.458 & $0.091 \%$ \\
6 & +25.672 & +0.263 & $1.026 \%$ & -0.111 & $0.433 \%$ \\
8 & -1.590 & -0.231 & $14.548 \%$ & +0.037 & $2.332 \%$ \\
10 & +0.111 & +0.002 & $2.217 \%$ & -0.058 & $51.947 \%$ \\
$\mathbf{1 2}$ & $\mathbf{- 0 . 0 0 9}$ & $\mathbf{+ 0 . 0 9 4}$ & $\mathbf{1 0 3 9 . 1 3 3 \%}$ & $\mathbf{+ 0 . 0 8 4}$ & $\mathbf{9 3 4 . 8 1 6 \%}$ \\
\hline
\end{tabular}

Note. Values in bold indicate the largest variation caused by the effect of the zonal winds.

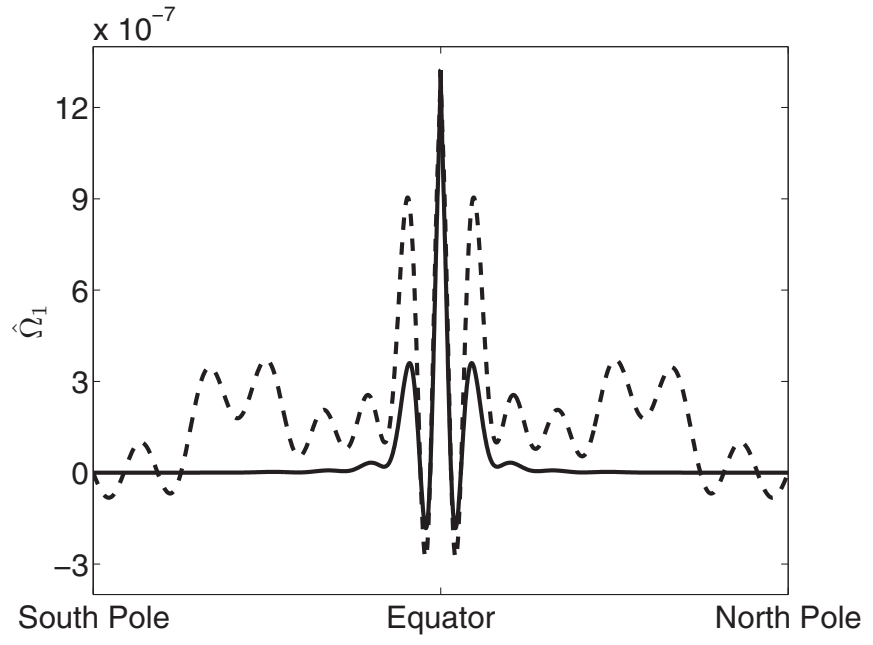

Figure 2. Profile of $\widehat{\Omega}_{1}$ as a function of the distance $s$ from the rotation axis. The dashed line for $D \rightarrow \infty$ corresponds to the observed Jovian zonal winds extending from the outer surface at all latitudes to the metallic molecular interface. The solid line for $D=0.1$ corresponds to the zonal winds confined within the equatorial region between the latitudes $\varphi= \pm 25^{\circ}$ with the maximum depth about $10 \% R_{e}$. This region contains $0.18 \%$ of Jupiter's total mass.

$r<0.78 R_{e}$ where the Jovian magnetic field is generated by the dynamo process and the outer molecular envelope $0.78 R_{e}<r<R_{e}$ into which the observed zonal winds might be able to penetrate. It is unlikely that the observed zonal winds can persist in the metallic core because of the strong magnetic braking there (Liu et al. 2008). Our model of Jupiter's interior is also assumed to comprise two parts: the spheroidal core in $0<\eta<0.78 R_{e}$ is rotating uniformly with $\Omega_{0} \hat{\mathbf{z}}$ while the spheroidal envelope in $0.78 R_{e}<\eta<R_{e}$, whose outer bounding surface has eccentricity $\mathcal{E}_{J}=0.3543$, has the zonal flow $\widehat{\Omega}_{1} \hat{\mathbf{z}} \times \mathbf{r}$ with the angular velocity $\left(\Omega_{0}+\widehat{\Omega}_{1}\right) \hat{\mathbf{z}}$ (Figure 1 ). Since our model is non-spherical, $\eta$ represents the radius in oblate spheroidal coordinates at the equatorial plane while the interface between the core and the envelope is marked by the density constant of an oblate spheroidal surface with eccentricity $\mathcal{E}_{I}=0.3202$. The zonal winds $\widehat{\Omega}_{1} \hat{\mathbf{z}} \times \mathbf{r}$ take only the north-south symmetric component of the observed zonal winds by fitting them to even harmonics up to degree 30 . In order to identify the gravitational signature produced only by the equatorial zonal jets, we construct a hypothetical profile of the internal angular velocity:

$$
\widehat{\Omega}_{1}(s, D)=\Omega_{1}(s) \mathrm{e}^{-\left(R_{e}-s\right) /\left(D R_{e}\right)},
$$

where $s$ denotes the distance from the rotation axis, $D$ is a decay factor measuring the confinement of the zonal winds to the equatorial region and $\Omega_{1}(s) \hat{\mathbf{z}} \times \mathbf{r}$ represents the observed zonal
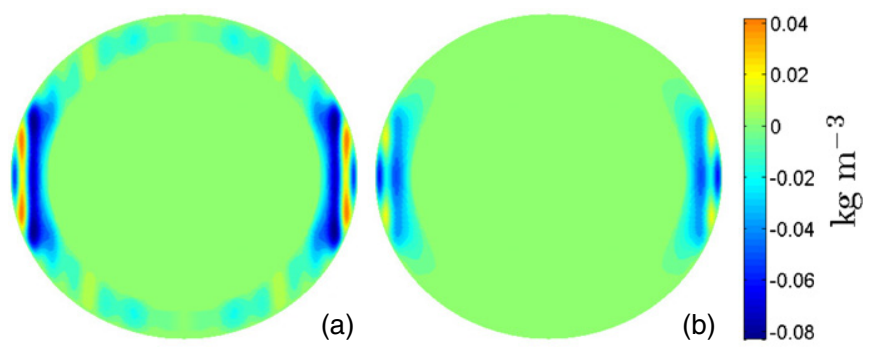

Figure 3. Contours of the density anomaly induced by the zonal winds in a meridional plane of the rotationally distorted Jupiter with the outer surface eccentricity $\mathcal{E}_{J}=0.3543$ for two different cases: (a) $D=\infty$ and (b) $D=0.1$.

winds that are equatorially symmetric and extend, parallel to the rotation axis, from the Jovian outer surface at all latitudes to the interface at $\eta=0.78 R_{e}$. When $D \rightarrow \infty$, we have $\widehat{\Omega}_{1}(s, \infty)=\Omega_{1}(s)$.

\section{RESULTS}

When $D=0.1$, the zonal winds are primarily confined within a small equatorial domain between the latitudes $\varphi= \pm 25^{\circ}$ with the maximum depth $10 \% R_{e}$ containing $0.18 \% M_{J}$. For the purpose of easy comparison, the profile of $\widehat{\Omega}_{1}(s, D)$ as a function of $s$ is displayed in Figure 2 for the two cases with $D \rightarrow \infty$ (the dashed line) and $D=0.1$ (the solid line). It can be seen that the zonal winds for $D=0.1$ are almost completely confined in the equatorial region. This special case is equivalent to an assumption that the zonal winds in the non-equatorial regions with the latitudes $25^{\circ} \leqslant \varphi \leqslant 90^{\circ}$ and $-90^{\circ} \leqslant \varphi \leqslant-25^{\circ}$ are restricted in a very thin outer layer and, thus, cannot produce any detectable gravitational signature. With the profile of the zonal winds $\widehat{\Omega}_{1} \hat{\mathbf{z}} \times \mathbf{r}$, we can compute the density anomalies induced by the winds, that are displayed in Figure 3 for two different cases with $D=\infty, 0.1$. Using the density anomalies, we can readily compute the correction $\Delta J_{n}$ caused by the zonal winds. Results are shown in Table 1.

We concentrate on the gravitational coefficient $\left(J_{12}+\Delta J_{12}\right)$ whose size is directly relevant to the effect of the Jovian zonal winds. If Jupiter did not have the zonal winds, i.e., $\widehat{\Omega}_{1}=0$ and $\Delta J_{n}=0$ for all $n$, the coefficient $\left(J_{12}+\Delta J_{12}\right)$ would be quite small with $J_{12}=-0.009 \times 10^{-6}$. When the zonal winds in the case $D=\infty$ fully penetrate the whole molecular envelope at all the latitudes, $\left(J_{12}+\Delta J_{12}\right)$, due to the redistribution of mass induced by the deep zonal winds everywhere, increases dramatically to $\left|\Delta J_{12} / J_{12}\right|=1039 \%$. This result is qualitatively consistent with the previous spherical-shell calculation (Kaspi et al. 2010). Note that only the total value $\left(J_{12}+\Delta J_{12}\right)$ can be measured. If $\left|\Delta J_{12} / J_{12}\right| \gg 1$, the wind-induced gravitational signature can be then easily isolated from the measurement. For the lower-order coefficients, such as $\left(J_{6}+\Delta J_{6}\right)$ which has been already accurately measured (Jacobson, JUP230 
orbit solution, http://naif.jpl.nasa.gov/pub/naif/JUNO/kernels/ spk/jup230.bsp.lbl(2003)), it would be difficult to identify the wind-related gravitational signature because $\left|\Delta J_{6} / J_{6}\right| \ll 1$, as shown in Table 1 . This is why the high-precision gravity measurement for the high-order coefficient $\left(J_{12}+\Delta J_{12}\right)$ offers hope of solving the puzzle about the depth of the Jovian zonal winds. When the zonal winds in the case $D=0.1$ are confined in the small equatorial region, $\left(J_{12}+\Delta J_{12}\right)$, due to the density anomalies induced only by the equatorial zonal jets, also increases dramatically to $\left|\Delta J_{12} / J_{12}\right|=935 \%$. In other words, the equatorial zonal jets confined in the latitudes $\varphi= \pm 25^{\circ}$ with the maximum depth $10 \% R_{e}$ containing $0.18 \%$ of the total mass of Jupiter make a surprising $90 \%$ contribution to the total gravitational coefficient $\left(J_{12}+\Delta J_{12}\right)$ that will be accurately measured by the Juno spacecraft. We have also carried out computations for the solutions with $0.1<D<\infty$ whose results lie expectedly between the two extreme cases $D=0.1$ and $D=\infty$; for instance, the case $D=0.5$ gives rise to $\left|\Delta J_{12} / J_{12}\right|=1026 \%$. There are two reasons why the equatorial jets dominate the contribution: the gravitational signal is sensitive to the location of the equatorial region in an oblate spheroid and the density anomalies are primarily driven by the wind shear $d \Omega_{1} / d s$ which is overwhelmingly predominant near the equator.

\section{CONCLUSIONS AND DISCUSSION}

The result of our models has a significant implication for the interpretation of the high-precision gravitational measurements. If the high-order gravitational coefficients were sensitive to the penetration depth of the zonal winds, an estimated extent of the zonal winds into the Jovian interior could be obtained by comparing the measured $\left(J_{12}+\Delta J_{12}\right)$ to the corresponding value from the accurate model computation using different depths. However, when the equatorial zonal jets-whose existence is unlikely to be strongly affected by either the Jovian magnetic field or the stable stratification (Liu et al. 2008; Jones \& Kuzanyan 2009; Gastine \& Wicht 2012)_make the predominant contribution, as shown in this study, the high-order gravitational coefficients like $\left(J_{12}+\Delta J_{12}\right)$ are largely independent of the penetration depth of the zonal winds in the non-equatorial regions with the latitudes $25^{\circ} \leqslant \varphi \leqslant 90^{\circ}$ and $-25^{\circ} \leqslant \varphi \leqslant-90^{\circ}$.

The Sun is a magnetic, slowly rotating star whose internal differential rotation is known and whose globally systematic magnetic variation, such as the 22 year sunspot cycle, is thought to be associated with the solar tachocline, a highly differentially rotating transition zone between the convection envelope and radiative core of the Sun (e.g., Parker 1993). Jupiter is a magnetic, rapidly rotating planet whose internal zonal-wind structure is currently unknown. If there exists a solar-type tachocline within the deep interior of Jupiter, which was not considered in the present study, the problem of interpreting the windinduced gravitational signature would be highly complicated. However, it is widely thought that the internal dynamics of Jupiter, in contrast to that of the Sun, is predominantly controlled by the effect of rotation (e.g., Busse 1976; Zhang 1992; Gastine \& Wicht 2012) and, hence, approximately described by the Proudman-Taylor theorem which states that infinitesimal steady motions in a rotating inviscid fluid are two dimensional with respect to the direction of the angular velocity. Of course, the theorem cannot directly apply to the flow within Jupiter which is not infinitesimal and concerns a fluid of non-zero viscosity. The theorem nevertheless correctly reveals a tendency for rapid rotation to inhibit the variation of the fluid motion along the axis of rotation. Consequently, the Jovian zonal winds, although their depth is currently unknown, are likely to depend essentially on the distance from the rotation axis. It follows that a solar-type tachocline is unlikely to exist in the deep interior of rapidly rotating Jupiter.

We have assumed in this study that the Jovian zonal winds are hemispherically symmetric and azimuthally axisymmetric. But the Jovian zonal winds, largely due to the Red Spot, are hemispherically non-symmetric and azimuthally non-axisymmetric and, consequently, contribute to the odd and non-axisymmetric gravitational harmonics. (Kaspi 2013) suggests that a deep hemispherically non-symmetric zonal wind would produce odd gravitational harmonics that might be detectable by Juno's measurements. Furthermore, if the Red Spot represents a deep phenomenon, the azimuthally non-axisymmetric gravitational harmonics would also be significant. Computation of the nonaxisymmetric harmonics caused by the Red Spot is mathematically more difficult and will be considered in a future study.

Saturn also has similar atmospheric circulations dominated by the equatorial zonal jets (Ingersoll 1990). Cassini spacecraft will orbit Saturn and accurately measure its gravitational field at the end of its mission. On the basis of our results for Jupiter, it seems reasonable to anticipate that the equatorial zonal jets of Saturn would also dominate the gravitational signals of the zonal winds and, thus, the size of its high-order gravitational coefficients would not reflect the penetration depth of the Saturn's zonal winds because they would be nearly independent of the depth of the Saturn's zonal winds in the non-equatorial regions.

X.L. is supported by NSFC/11133004 and CAS under grant numbers KZZD-EW-01-3 and XDB09000000, K.Z. is supported by UK NERC and STFC grants and G.S. is supported by the National Science Foundation under grant NSF AST-0909206. The parallel computation is supported by the Shanghai Supercomputer Center.

\section{REFERENCES}

Atkinson, D. H., Pollack, J. B., \& Seiff, A. 1996, Sci, 272, 842

Bolton, S. J. 2005, in Juno Final Concept Study Report, Tech. Rep. AO-03OSS-03, New Frontiers (Washington, DC: NASA)

Busse, F. 1976, Icar, 29, 255

Chandrasekhar, S. 1933, MNRAS, 93, 390

Gastine, T., \& Wicht, J. 2012, Icar, 219, 428

Guillot, T. 2005, AREPS, 33, 493

Gundlach, J. H., \& Merkowitz, S. M. 2000, PRL, 85, 2869

Heimpel, M., Aurnou, J., \& Wicht, J. 2005, Natur, 438, 193

Hubbard, W. B. 1974, AZh, 51, 1052

Hubbard, W. B. 1999, Icar, 137, 357

Ingersoll, A. P. 1990, Sci, 248, 308

Ingersoll, A. P., \& Cuzzi, J. N. 1969, JAtS, 26, 981

Jones, C. A., \& Kuzanyan, K. M. 2009, Icar, 204, 227

Kaspi, Y. 2013, GeoRL, 40, 676

Kaspi, Y., Hubbard, W. B., Showman, A. P., \& Flierl, G. R. 2010, GeoRL, 37, L01204

Kong, D., Liao, X., Zhang, K., \& Schubert, G. 2013a, Icar, 226, 1425

Kong, D., Zhang, K., \& Schubert, G. 2012, ApJ, 748, 143

Kong, D., Zhang, K., Schubert, G., \& Anderson, J. 2013b, ApJ, 763, 116

Lian, Y., \& Showman, A. P. 2010, Icar, 207, 373

Limaye, S. S. 1986, in Jovian Atmospheres, ed. M. Allison \& L. D. Travis, Vol. 2441 (New York: NASA Conf. Pub.), 262

Liu, J., Goldreich, P. M., \& Stevenson, D. J. 2008, Icar, 196, 653

Nettelmann, N., Becker, A., Holst, B., \& Redmer, R. 2012, ApJ, 750, 52

Null, G. W. 1976, AJ, 81, 1153

Parker, E. 1993, ApJ, 408, 707

Porco, C. C., West, R. A., McEwen, A., et al. 2003, Sci, 299, 1541

Seidelmann, P. K., Archinal, B. A., A'hearn, M. F., et al. 2007, CeMDA, 98,155

Stevenson, D. 1982, AREPS, 10, 257

Zhang, K. 1992, JFM, 236, 535

Zhang, K. 1993, JFM, 248, 203 